\title{
Documentos digitales y legislación archivística: el caso de México y Cuba
}

\author{
Yorlis Delgado López*
}

Artículo recibido:

2 de julio de 2021

Artículo aceptado:

6 de octubre de 2021

Artículo de investigación

\section{Resumen}

El patrimonio documental de una nación tiene un fuerte componente de los documentos digitales que se generan hoy en la administración pública. La gestión, el tratamiento y el acceso a esta especificidad documental son objeto de regulación y protección por el Derecho. Las legislaciones archivísticas de América Latina tienen en estos soportes un reto ineludible. México (2018) y Cuba (2020) son los cuerpos legales más contemporáneos y ya evocan estos registros. Con esta premisa, el objetivo de este artículo es analizar comparativamente las regulaciones jurídicas de los documentos digitales, a partir la legislación archivística de México y Cuba, con el fin de obtener apreciaciones conclusivas que sirvan de norma para el territorio latinoamericano.

* Colegio San Gerónimo de La Habana, Universidad de La Habana, Cuba

$$
\text { yorlis.delgad082@gmail.com }
$$

INVESTIGACIÓN BIBLIOTECOLÓGICA, vol. 36, núm. 90, enero/marzo, 2022, México, ISSN:
2448-8321 pp. 119-132 
La metodología y las técnicas empleadas fueron las inherentes al campo teórico-jurídico, el derecho comparado, analítico-deductivo y la técnica de análisis de documentos jurídicos. Se obtuvo una comparación valorativa de ambos corpus en cuanto a este objeto de protección y se infieren recomendaciones en este sentido para Latinoamérica. Se concluyó que los documentos digitales son parte indisoluble del patrimonio documental; la posición mexicana y cubana de protección legal del documento digital y su archivo, en espíritu normativo, es no contemplar el soporte.

Palabras clave: Documentos Digitales; Gestión Digital; Preservación Digital; Legislación Archivística

\section{Digital documents and Archival Legislation: the case of Mexico and Cuba \\ Yorlis Delgado López}

\section{Abstract}

The Documentary Heritage of a nation has a strong component of digital documents that is generated today in public administration. Its management, treatment and access to this documentary specificity are subject to regulation and protection by law. The archival laws of Latin America have in these supports an inescapable challenge. Mexico (2018) and Cuba (2020) are the most contemporary legal bodies and they already evoke these records. With this premise, the objective of this article is to comparatively analyze the legal regulations of digital documents, based on the Archival Legislation of Mexico and Cuba, in order to obtain conclusive assessments that serve as a norm for the Latin American territory. The methodology and techniques used were those inherent to the theoretical-legal field, comparative, analyticaldeductive and technical analysis of legal documents. An evaluative comparison of both corpus was obtained regarding this object of protection and recommendations are inferred, in this sense for Latin America. It was concluded that digital documents are an indissoluble part of the Documentary Heritage; the Mexican and Cuban position of legal protection of the digital document and its archive, in a normative spirit, is not to contemplate the support.

Keywords: Digital Documents; Digital Management; Digital Preservation; Archival Legislation 


\section{INTRODUCCIÓN}

— $\mathrm{n}$ los últimos años, los documentos o registros digitales han generaEdo mucha atención desde los estudios teóricos (Robledo, 2016; Álvarez, 2013: 7-12; Kroth y Flores, 2018). Es una apreciación lógica, pero hay una realidad que subyace: los registros digitales han invadido la Administración pública y han llegado para perdurar en el tiempo como parte del patrimonio documental de los países. En este sentido, se debe profundizar desde todas las áreas del conocimiento para lograr un ámbito objetivo de legado a las futuras generaciones de estos soportes. Desde el derecho es importante significar que la protección jurídica del documento, como elemento general, es fundamental de la legislación archivística como área del conocimiento. Es el bien jurídico esencial en esta especificidad y es imprescindible para configurar todo el ordenamiento jurídico que se desprende. Se sustenta en su carácter histórico inferido por su capacidad inclusiva de poder ser un elemento probatorio y testifical sobre determinados hechos o fenómenos sociales.

El valor de los documentos está presente en cualquiera de sus fases del ciclo de vida. Se insiste en que el recurso de salvaguarda no debe alcanzar sólo los documentos considerados históricos en un Estado, sino a toda la documentación generada por la Administración pública. Se asegura así que cumpla con el ciclo de vida de manera natural y el registro se enfrente a los procesos archivísticos imprescindibles para su gestión. Se debe enfocar el asunto de manera sistémica y holística, tratando de salvaguardar lo más importante de la masa documental que se genera en cualquier Estado (Delgado, 2021: 8).

En relación con la magnitud del tema y la necesidad de protección de este preciado bien, Pérez (1997: 55) alude que "se viene considerando al documento como la memoria de la Sociedad que describe, como la retentiva de la vida pública y privada”.

El razonamiento integral que hace Palma (2013) sobre el argumento da una medida del valor social y objetivo de este bien jurídico. Al respecto refiere que "los valores sociales podrán impulsar lo siguiente: potenciar la comprensión del pasado de la humanidad, permitir entender el presente y dilucidar el futuro, construir identidad, proteger raíces culturales, tomar conciencia, ejercer democracia, impulsar el desarrollo, realizar constante ejercicio crítico-creativo, entre otros aspectos” (Palma, 2013: 35-36). 
Delgado (2021: 8) ha referido que

Un elemento importante a regular se presenta a la hora de definir documento pues, amén de otros elementos configurativos, se debe asumir una postura amplia y no objetiva en cuanto al soporte en que se puede presentar el registro. El espíritu normativo no debe ir en función de restringir las formas que el soporte puede adoptar, siempre con una perspectiva muy amplia del asunto. Esto se traduce en que se puede presentar en piedra, papiro, metal, papel, digital u otro que el futuro pueda surgir y que no se haya percibido aún. Este análisis es sumamente importante, se reitera, pues todos los demás preceptos que se regulan, relativos al tratamiento, valoración y acceso documental, le serán aplicables al registro público sin importar su formato.

La reflexión es inherente a la protección de los documentos en digital, de manera específica. Delgado (2021) considera un error desconocer este tema en la actualidad, pues se dejaría sin protección, en la práctica, a miles de expedientes digitales que devienen en históricos y que quedan a la merced de una depuración sin control por las organizaciones. Por ello se insiste en que en la medida que se logre adecuar las disposiciones jurídicas, se debe lograr una protección veraz y oportuna del registro. En tal sentido se podrá enfocar, desde la legislación, un mayor grupo de procesos archivísticos, también objeto de protección y que de manera necesaria deben impactar sobre el documento digital.

Se afirma, en consecuencia, derivado del análisis de las propias legislaciones y la práctica archivística, que los documentos digitales forman parte del patrimonio documental de un país. Su gestión, tratamiento, preservación y acceso son objeto de regulación y protección por el derecho. Las legislaciones archivísticas de América Latina tienen estos soportes un reto ineludible. México (2018) y Cuba (2020) son los cuerpos legales más contemporáneos y ya evocan estos registros desde la norma superior, con un enfoque primario, pero sin desconocerlos. Otros países como Colombia y Brasil han optado por normas complementarias para tratar lo concerniente al documento digital.

El objetivo de este artículo es analizar comparativamente las regulaciones jurídicas de los documentos digitales a partir la legislación archivística de México y Cuba, a fin de obtener apreciaciones conclusivas que sirvan de norma para el territorio latinoamericano. La metodología y las técnicas empleadas fueron las inherentes al campo teórico-jurídico, que permitió dejar asentado el ámbito conceptual de forma analítica y comparativa dentro del cual discurren las regulaciones de los documentos digitales en México y Cuba; el derecho comparado, que facilitó la comparación entre los corpus normativos de ambas naciones; el método analítico-deductivo, que compulsó inducir las concepciones doctrinales que giran alrededor al tema en ambos países y 
asumir consideraciones y recomendaciones relativas al ámbito de regulación jurídica, y la técnica de análisis de documentos jurídicos, que facilitó el estudios de las normas en Cuba y México.

Se obtiene entonces una comparación valorativa de ambos corpus en cuanto a este objeto de protección, teniendo en cuenta indicadores establecidos por el autor. Además, se infieren un grupo de elementos que se recomiendan sean considerados en otras leyes nacionales.

\section{UNA REFLEXIÓN SOBRE EL TRATAMIENTO ARCHIVÍSTICO DE LOS} DOCUMENTOS DIGITALES

Este análisis es expresamente jurídico. No obstante, es necesario valorar algunos criterios desde las ciencias archivísticas que se manejan para su ulterior comprensión. La papelería digital ha inspirado en los últimos años numerosos estudios relativos a su gestión, preservación y acceso. La mayoría de los teóricos (Robledo, 2016; Álvarez, 2013: 7-12; Kroth y Flores, 2018), incluido el autor, consideran que tiene determinadas particularidades en cuanto a su gestión y conservación. En tal sentido, sólo cambian las condiciones con que se conservarían estos registros. Implicaría mayores recursos económicos para comprar la estantería adecuada, mantener los valores de temperatura y humedad, el equipamiento para ver el contenido de lo que está registrado, etcétera. Países como Japón y China ya tienen en sus archivos nacionales secciones dedicadas a conservar este tipo de documentos, con las condiciones necesarias para preservarlos por un largo periodo de tiempo. En Iberoamérica se aprecian proyectos como Interpares (Barnard, 2020) y otros que ya muestran resultados prometedores. Sin embargo, se aprecian más similitudes en cuanto a su gestión y tratamiento archivístico se refiere, aplicándose los mismos principios y normas que la archivística establece para el resto de los documentos similares. Se entiende que ha cambiado el formato y no el registro de información. La circunstancia definida adquiere relevancia en términos jurídicos, pues se entiende que sólo se establecerán las reglas específicas para tratar este documento. No se desconocen algunas peculiaridades en cuanto a su gestión propiciadas por las propias características del formato, pero en principio y teoría su aplicación es muy similar.

Se aprecian similitudes, además, en torno a los procederes relativos al acceso a la información contenida en el registro digital. Este derecho, personalísimo e inalienable, no distingue en el formato en que se presente el documento. Se recuerda que el Estado está en la obligación de poner la información pública en función para el uso del ciudadano y no debe discriminarla, ni obviar ninguna por el tipo de soporte (Nahabetián, 2010: 38-53). 
Al iniciar el análisis es necesario reflexionar sobre el objeto del mismo. Es menester entender que no se refiere a los miles de copias electrónicas generadas por los procesos de digitalización ${ }^{1}$ que se desarrollan en los archivos, pues en última instancia siempre quedarán los originales como muestra evidente de su existencia. Se precisa que, en este caso, la gran preocupación serían los registros que jamás llegan al papel y que son tan importantes para la gestión y para reconstruir trámites y procederes organizacionales.

Para el análisis comparativo se escogen los dos corpus normativos relativos a archivos más contemporáneas en el área de Latinoamérica: México (2018) y Cuba (2020). Se desarrolla independientemente de los sistemas políticos y el rango legislativo de las disposiciones con el sumo interés sobre las normas establecidas y su impacto sobre la gestión documental. Con ello se pretende inducir algunas recomendaciones en el orden regulatorio con vistas a futuras normativas. Se han preestablecido por el autor un grupo de indicadores para propiciarlo e inducir los resultados:

a) Definición legal: general y específica.

b) Obligaciones específicas con la documentación digital.

c) Regulaciones a la preservación digital.

\section{Tratamiento jurídico a los documentos digitales en México}

La Ley General de Archivos de los Estados Unidos Mexicanos del 13 de junio de 2018 (AGN, 2019) ${ }^{2}$ se infiere como la supra normativa que regula el actuar archivístico en ese país. Es muy interesante que desde los objetivos de la disposición hay una intención de promover el desarrollo archivístico digital y la creación de un sistema de archivos digitales como las bases para crear un gobierno digital con el objetivo supremo de prestar servicios a usuarios ${ }^{3}$ (AGN, 2019).

1 "Digitalizar" se refiere a la conversión de un soporte físico en una copia digital, usualmente por medio de escaneo u otro proceso de captura de imágenes; en este sentido, es importante hacer la distinción entre los documentos digitalizados, creados a partir de un archivo físico, y los documentos que "nacieron digitales" (AGN, 2015:3).

2 Ver https://www.alaarchivos.org/normatividad-archivistica/ para consultar normatividad archivística adicional.

3 "Artículo 2. Son objetivos de esta Ley: [...] V. Sentar las bases para el desarrollo y la implementación de un sistema integral de gestión de documentos electrónicos encaminado al establecimiento de gobiernos digitales y abiertos en el ámbito federal, estatal y municipal que beneficien con sus servicios a la ciudadanía." (AGN, 2019) 
La normativa regula un grupo de definiciones generales para su entendimiento a derecho que propician el entendimiento de su estudio. En su artículo 4 define documento de archivo como "A aquel que registra un hecho, acto administrativo, jurídico, fiscal o contable producido, recibido y utilizado en el ejercicio de las facultades, competencias o funciones de los sujetos obligados, con independencia de su soporte documental" (AGN, 2019).

Queda clara la negación del soporte documental como elemento configurativo de esta definición, dejando a otros esta función. Sin embargo, resulta trascendental que no dejó a interpretaciones qué se entendería por soporte documental, definiéndolo ${ }^{4}$ y preestableciendo al citar el digital entre ellos. Es una clara apreciación y muy importante, pues todas las regulaciones en el orden de gestión, preservación y acceso que en lo sucesivo se enumeran le son aplicables a estos documentos en este soporte.

En relación al objeto de estudio, la ley mexicana no definió un concepto específico de este particular; sin embargo, reguló qué se entendería por expediente electrónico: "conjunto de documentos electrónicos correspondientes a un procedimiento administrativo, cualquiera que sea el tipo de información que contengan". (AGN, 2019: art. 4, XXX). Es decir, se estableció específicamente qué se entiende por este supuesto.

Relativo a las obligaciones específicas con la documentación digital, se reitera que la legislación mexicana da un concepto jurídico de documento de archivo donde el soporte no juega un papel imperativo, por lo que todas las obligaciones establecidas le son aplicables a los documentos digitales. Sin embargo, se regulan un grupo de obligaciones para los sujetos obligados a cumplir la disposición en el "Capítulo IX. De los documentos de archivo electrónicos” (AGN, 2019: arts. 41-49). Se establecen obligaciones a:

- Contemplar para la gestión electrónica de documentos "la incorporación, asignación de acceso, seguridad, almacenamiento, uso y trazabilidad" (AGN, 2019: art. 41).

- Establecer en un "programa anual los procedimientos para la generación, administración, uso, control y migración de formatos electrónicos" (AGN, 2019: art. 42).

- Aprobar "planes de preservación y conservación de largo plazo que contemplen la migración, la emulación o cualquier otro método de preservación y conservación de los documentos de archivo electrónicos" (AGN, 2019: art. 42).

4 "LIV. Soportes documentales: A los medios en los cuales se contiene información además del papel, siendo estos materiales audiovisuales, fotográficos, fílmicos, digitales, electrónicos, sonoros, visuales, entre otros." (AGN, 2019: art. 4) 
- Adoptar medidas de organización y tecnológicas "para garantizar la recuperación y preservación de los documentos de archivo electrónicos producidos y recibidos que se encuentren en un sistema automatizado para la gestión documental y administración de archivos, bases de datos y correos electrónicos a lo largo de su ciclo vital" (AGN, 2019: art. 44).

- "Implementar sistemas automatizados para la gestión documental y administración de archivos" (AGN, 2019: art. 45).

- Garantizar "la validez jurídica de los documentos de archivo electrónico, los sistemas automatizados para la gestión documental y administración de archivos y la firma electrónica avanzada de la obsolescencia tecnológica mediante la actualización, de la infraestructura tecnológica y de sistemas de información que incluyan programas de administración de documentos y archivos, en términos de las disposiciones jurídicas aplicables" (AGN, 2019: art. 49).

Con las especificidades descritas, México regula el archivo y preservación de los documentos digitales. De una manera sutil se induce la necesidad de que los sujetos obligados presenten la debida atención a estos registros y se adopten las medidas señaladas, no sólo para su preservación sino para su gestión y acceso a los interesados.

Relativo a la preservación digital, el propio Capítulo IX de la normativa regula la obligación de los sujetos a establecer planes de preservación a largo plazo y refuerza la necesidad de tener en cuenta los procederes migratorios o cualquier otro método efectivo para lograr este fin (AGN, 2019). El artículo 43 insiste en la necesidad de configurar "en el programa anual la estrategia de preservación a largo plazo de los documentos de archivo electrónico y las acciones que garanticen los procesos de gestión documental electrónica" (AGN, 2019). Incluso en el artículo 62 recomienda una clara inducción al uso de un servicio de nube como plataforma de gestión, preservación y acceso a los documentos que por su valor así lo ameriten (AGN, 2019).

De esta manera, la disposición supra en el orden archivístico en México defiende y protege sus documentos digitales como un soporte al que ya la legislación no puede desconocer y dar un tratamiento general. El autor considera que las normas antes mencionadas dan una protección integral y sistémica a estos registros de manera que no sólo se infiera su preservación, sino que se comprenda el valor de uso que éstos poseen para el ulterior desarrollo de las investigaciones y la sociedad en sentido general. 


\section{Tratamiento jurídico de los documentos digitales en Cuba}

El Decreto Ley No. 3/2020 "Del Sistema Nacional de Gestión Documental y Archivos de la República de Cuba" es la disposición superior en la materia objeto de este estudio. Sus normas complementarias (Decreto 7/2020 "Reglamento del Sistema Nacional de Gestión Documental y Archivos de la República de Cuba", Resolución 201/2020 "Lineamientos Generales para la Conservación de las Fuentes Documentales de la República de Cuba" y la Resolución 202/2020 "Lineamientos Generales para la Digitalización de las Fuentes Documentales de la República de Cuba", ambas del Ministerio de Ciencia, Tecnología y Medio Ambiente) dan una protección integral a los archivos y documentos, marcando así pautas para la protección de la memoria histórica cubana. Esta disposición es la más contemporánea en Latinoamérica entre las de su clase.

De manera general, el Decreto Ley No. 3/2020 reguló qué se entendería por documento de archivo y estableció en su artículo 9.2 (cursivas propias) que

El documento de archivo es el medio en el que se registra o se trasmite información en soportes como papel, digital, fotográfico, fílmico, audiovisual, radiofónico y fonográfico, refleja además el testimonio material realizado por una persona natural o jurídica en el ejercicio de sus funciones o su actividad intelectual, proporciona evidencia de actos y transacciones administrativas y por su valor debe ser objeto de conservación, excepto las publicaciones periódicas y bibliográficas.

Esta disposición se afilió al criterio de mencionar tácitamente los tipos de soportes en que se puede presentar el documento. Esto pudiera considerarse un retroceso pragmático después de que el Decreto Ley No. 265/2009, norma que antecedió a la legislación vigente, superó esta redacción y la desarrolló de manera más general y abierta, incluso al surgimiento de nuevos soportes. Se entiende que es el reflejo del nivel de conocimientos sobre archivística imperante entre dirigentes y funcionarios.

Se reitera la importancia de la definición mencionada en el nuevo contexto. Una interpretación extensiva de la norma da la medida que los registros digitales cumplen los parámetros establecidos en el ciclo de vida de los documentos, igual que los demás, y se aplican sobre ellos los mismos principios de gestión, valoración, tratamiento documental y acceso.

Resulta redundante que en el capítulo VI, "De las aplicaciones informáticas y la administración de documentos electrónicos", aparece una definición 
especifica relativa a los documentos digitales ${ }^{5}$ que ratifica explícitamente esta idea. En la regulación de los tipos de archivos aceptados por esta legislación aparecen los archivos especiales, en cuya definición ${ }^{6}$ y contexto de actuación es evidente que influye de manera determinante el formato de los documentos que conserva y la recurrencia de condiciones especiales de conservación. Aquí cabían perfectamente los archivos digitales, pero sólo se contemplaron los objetos producto de la digitalización.

El Decreto Ley No. 3/2020 no estableció obligación específica alguna para las entidades cubanas sobre los registros digitales; sólo reguló las consecuencias que se derivan del cumplimiento de la disposición. Tampoco reguló pretexto alguno que se pueda vincular con la preservación digital. Específicamente, y a juicio del autor, de una manera más didáctica y ajustada a lo que en materia jurídica compete, la Resolución 201/2020 “Lineamientos Generales para la Conservación de las Fuentes Documentales de la República de Cuba” reguló toda una tecnología para lograr su fin. Una evidente muestra de que, aún en países sin recursos, se pueden aprobar medidas de preservación digital. En las normas específicas de los Lineamientos se sancionan un grupo de requerimientos formales según el soporte en que se puede presentar el documento digital (Resolución 201/2020: arts. 18-20).

La legislación archivística cubana logró normalizar los procesos de digitalización de documentos (Resolución 202/2020: art. 2), procederes tan necesarios y oportunos cuando de conservación y acceso se habla. Sobre todo, al pensar que los recursos tan limitados de una nación subdesarrollada se emplearán a tenor de una base operativa de centralización de prioridades que limitará la duplicidad y traerá mayor operatividad y eficiencia en este actuar.

A pesar del tratamiento legal dado a este tema en Cuba, se considera insuficiente, teniendo en cuenta el nivel de los ejecutores de la norma y la apremiante situación de pérdidas del patrimonio digital cubano. Sigue observándose, a contraluz, un punto vulnerable en este tema y en el cual se debe incidir rápidamente: los ejecutores o sujetos obligados a proteger el patrimonio documental

5 "Artículo 62. El documento digital de archivo es el registro de información que se genera, recibe, almacena y comunica por medios digitales, durante todo su ciclo de vida, producida por una persona natural o jurídica en razón de sus actividades o funciones, que tiene valor administrativo, fiscal, legal, científico, histórico, técnico o cultural y se trata conforme a los principios y procesos archivísticos." (Decreto Ley No. 3/2020.)

6 "Artículo 48.1. Los archivos especiales son aquellos que se integran por documentos que emplean un lenguaje diferente al textual, para trasmitir información y requieren de condiciones especiales de instalación y conservación.

"2. Se consideran documentos en soportes especiales los fotográficos, fílmicos, audiovisuales, radiofónicos, fonográficos, y los artefactos y objetos digitalizados.

"3. Las normas metodológicas para la conservación, tratamiento y difusión de los documentos en soportes especiales, se regulan en las disposiciones complementarias a este Decreto-Ley.” 
no ven en el soporte digital un recurso importante, a pesar de que, tal como se analizó, la disposición lo contempla.

\section{Puntos Que Pueden SER CONSIDERADOS EN OTRAS Leyes NACIONALES}

El Anexo resume los principales criterios de la comparación hecha en torno a los documentos digitales en la legislación archivística de México y Cuba. Después de su análisis y valoración se deducen algunas recomendaciones a futuro para disponer sobre el tema. Se recuerda que la legislación archivística es una herramienta y por consecuente su gestión debe encaminarse siempre a resolver problemas prácticos y objetivos del quehacer archivístico de los países. En tal sentido y después del análisis que antecede, sólo se presumen estas recomendaciones generales sin llegar a una apreciación más específica del fenómeno, pues ello dependerá siempre de cada territorio y sus necesidades archivísticas y de gestión:

- En primer orden, es ineludible que se regule el tema de objeto de análisis. La especificidad y la primacía de estos documentos hacen que la legislación no pueda desconocerlos. Se reitera que muchos registros digitales tienen un valor documental indiscutible y su pérdida, deterioro o alteración ocasionará grandes daños, irreparables en la mayoría de los casos, al patrimonio documental de los países en cuestión.

- Establecer definiciones generales del bien jurídico documento en las que el soporte documental no sea imprescindible para su configuración, de manera que el documento digital sea objeto de todas las regulaciones en cuanto a gestión, preservación y acceso que se establezcan a posteriori.

- Regular definiciones específicas que infieran el valor de uso del documento digital.

- Regular de forma específica las obligaciones de las entidades referidas al deber sobre la gestión, preservación y acceso como único medio para el ulterior uso de los documentos digitales. Aunque algunos procederes sean similares se entiende que es preciso regular la especificidad de las características de la preservación digital, como las normas generales de la gestión específica, las obligaciones y los límites del deber hacer de los sujetos obligados a cumplir las disposiciones archivísticas, por sólo citar algunas. 


\section{Conclusiones}

El análisis de los elementos concernientes al tratamiento jurídico de los documentos digitales en las legislaciones archivísticas más contemporáneas de Latinoamérica (Cuba y México) lleva a las siguientes reflexiones:

- Los documentos digitales son parte indisoluble del patrimonio documental y les son aplicables los principios que la archivística y la legislación específica prevé en cuanto a su gestión, procesamiento, transferencia, valoración y acceso a su información.

- Muy novedoso resulta el tratamiento legal de los documentos en soporte electrónicos, regulando para ellos la obligación de conservar de manera permanente a los sujetos de la ley; un marco de referencia y comparación necesario.

- La posición mexicana y cubana de protección legal del documento digital y su archivo, en espíritu normativo, es no contemplar el soporte como elemento configurativo de la definición sustancial del bien y enfocarse en otros elementos más importantes para la configuración del concepto.

- Ambas disposiciones regulan el bien documentos digitales de una manera general, y propician la gestión y el acceso. En ambos casos la praxis demuestra que las tecnologías de regulación son perfectibles y ajustables.

- Las recomendaciones legislativas sobre el tratamiento legal de los archivos digitales pretenden lograr un sistema de protección legal que sea realmente sea efectivo y funcional en la praxis. Es esta la encomienda principal del ordenamiento específico.

\section{REFERENCIAS}

Álvarez, Ascanio. 2013. "Perspectivas actuales sobre la autenticidad y autentificación de los documentos electrónicos de archivo". Boletín del Archivo Nacional (21): 7-26. AGN (Archivo General de la Nación de México). 2015. Recomendaciones para proyectos de digitalización de documentos. México: AGN. https://www.gob.mx/cms/uploads/attachment/file/146401/Recomendaciones_ para_proyectos_de_digitalizacion_de_documentos.pdf

AGN. 2019. Ley General de Archivos. México: Cámara de Diputados, Diario Oficial de la Federación de 15 de junio de 2018. http://www.diputados.gob.mx/LeyesBiblio/ref/lga.htm 
Barnard, Alicia. 2020. El proyecto InterPARES en América Latina y el Caribe. Apuntes sobre archivos digitales, transparencia, acceso a la información y protección de datos personales. México: Inai.

https://inai.janium.net/janium/Documentos/3801/InterPARES.pdf

Decreto 7/2020. "Reglamento del Sistema Nacional de Gestión Documental y Archivos de la República de Cuba”. Gaceta Oficial de la República de Cuba 55, edición ordinaria. 31 de julio de 2020.

Decreto Ley No. 3/2020. "Del Sistema Nacional de Gestión Documental y Archivos de la República de Cuba”. Gaceta Oficial de la República de Cuba 55, edición ordinaria. 31 de julio de 2020.

Delgado, Yorlis. 2021. "Legislación Archivística: reflexiones doctrinales”. Revista de la Facultad de Derecho (50): 1-21. http://revista.fder.edu.uy/index.php/rfd/article/view/792

Kroth, Marcelo Lopes y Daniel Flores. 2018. "Autenticidad de documentos archivísticos digitales: análisis de un proceso de permisos y licencias". BIBLIOS, Revista de Bibliotecología y Ciencias de la Información (72): 67-79.

http://biblios.pitt.edu/ojs/index.php/biblios/article/view/361

Nahabetián, Laura. 2010. Acceso a la información pública: Pilar fundamental del buen gobierno. Montevideo, Uruguay: Amalio M Fernández.

Palma, Juan Miguel. 2013. "Valores sociales y valores patrimoniales: elementos para determinar la significación del patrimonio documental”. Biblioteca Universitaria (16): $33-45$.

https://doi.org/10.22201/dgb.0187750xp.2013.1.18

Pérez, Enrique. 1997. El archivo y el archivero. Islas Canarias, España: Canaricard.

Resolución 201/2020. "Lineamientos Generales para la Conservación de las Fuentes Documentales de la República de Cuba”. Cuba: Citma (Ministerio de Ciencia, Tecnología y Medio Ambiente). Gaceta Oficial de la República de Cuba 55, edición ordinaria, 31 de julio de 2020.

Resolución 202/2020. "Lineamientos Generales para la Digitalización de las Fuentes Documentales de la República de Cuba”. Cuba: Citma (Ministerio de Ciencia, Tecnología y Medio Ambiente). Gaceta Oficial de la República de Cuba 55, edición ordinaria, 31 de julio de 2020.

Robledo, Hugo R. 2016. "La gestión de documentos electrónicos en Repositorio Digital Institucional”. Revista REDar (2): 51-57.

http://redarchiveroscordoba.com/wp-content/uploads/2017/02/REDar-n\%C2\%B0-2-a\%C3\%B1o-1-julio-diciembre-2016.pdf

Para citar este texto:

Delgado López, Yorlis. 2022. "Documentos digitales y legislación archivística: el caso de México y Cuba”. Investigación Bibliotecológica: archivonomía, bibliotecología e información 36 (90): 119-132. http://dx.doi.org/10.22201/iibi.24488321xe.2022.90.58496 
Anexo. Tabla resumen de la comparación legislativa archivística de Cuba y México

\begin{tabular}{|l|l|l|}
\hline \multicolumn{1}{|c|}{ Indicadores } & \multicolumn{1}{|c|}{ Legislación archivística de México } & \multicolumn{1}{c|}{$\begin{array}{c}\text { Legislación archivística } \\
\text { de Cuba }\end{array}$} \\
\hline $\begin{array}{l}\text { Definición legal: } \\
\text { general y específica }\end{array}$ & $\begin{array}{l}\text { General: no tiene en cuenta el soporte } \\
\text { documental }\end{array}$ & $\begin{array}{l}\text { General: no tiene en cuenta el } \\
\text { soporte documental }\end{array}$ \\
\cline { 2 - 3 } & $\begin{array}{l}\text { Específica: se define sólo expediente } \\
\text { electrónico }\end{array}$ & $\begin{array}{l}\text { Específica: se define documen- } \\
\text { tos digitales }\end{array}$ \\
\hline $\begin{array}{l}\text { Obligaciones específicas } \\
\text { con la documentación } \\
\text { digital }\end{array}$ & Se regulan obligaciones específicas & $\begin{array}{l}\text { No se regulan obligaciones } \\
\text { específicas }\end{array}$ \\
\hline $\begin{array}{l}\text { Regulaciones a la } \\
\text { preservación digital }\end{array}$ & $\begin{array}{l}\text { Obligación de aprobar planes y programas } \\
\text { de preservación a largo plazo }\end{array}$ & $\begin{array}{l}\text { Normas específicas de preserva- } \\
\text { ción digital }\end{array}$ \\
\hline
\end{tabular}

